\title{
Computational Musicology for Raga Analysis in Indian Classical Music: A Critical Review
}

\author{
Kunjal Gajjar \\ Ahmedabad University \\ Ahmedabad -380009 \\ Gujarat, India
}

\author{
Mukesh Patel \\ Charotar University of Science \\ and Technology \\ Changa, Gujarat, India
}

\begin{abstract}
The paper gives brief introduction of Computational Musicology as a sub field of Artificial Intelligence (AI). It explains evolution of computational Musicology field and existing work done in the context of Western and Indian Music. It also compares Western Music with Indian Classical Music (ICM) and points out essential differences between them, illustrating how analysis of Western Music is not compatible with Indian Classical Music. A section in paper also gives introduction to Hindustani Classical Music and describes features that determine the grammar for representation and analysis of a raga. Section 5 represents the study of existing work in the field of Computational Musicology for ICM and identifies limitations and advantages of the methods.
\end{abstract}

\section{General Terms}

Pattern Recognition, Electronic Music Distribution, Computational Musicology, Artificial Intelligence

\section{Keywords}

Computational Musicology, Indian classical music (ICM), swars, Raga, Hindustani Classical Music, Note Transcription, Raga analysis

\section{INTRODUCTION}

Computational Musicology is a subfield of Artificial Intelligence (AI) which deals with music and computation. Its aim is to develop expert systems for the music domain which can recognize music, generate it and can also be used for learning music [3]. It is defined as the study of music with computational modeling and simulation [2].

This new interdisciplinary research area which requires the knowledge of music and computer science was initiated in 1960s by Heckman. It focused on the use of computers for analyzing complex and vast information available in the music domain [1]. Several mathematical and statistical methods are used along with the complex algorithms for accomplishing tasks such as music analysis, music composition, pattern recognition, genre classification and Music Information Retrieval [6].

Since 1960's there has been reasonably good amount and good quality work in computational musicology for various genres of Western music. However, Indian music genres, particularly classical music though of great antiquity have been relatively unexplored in terms of computational musicology [14] [16].

The uniqueness of Indian Classical Music (ICM) is in its melodic structure which is based on the raga system which is described in more detail in Section 1.3. Most of the computational musicology research for ICM has largely focused on analysis of the audio waves to recognize/identify or classify raga. However, very few studies have attempted to investigate the structures of raga or have reported any representation of ICM [11]. The main reason for this is that unlike Western Classical Music, ICM raga structure is not fixed, nor does it exist in a written form. Instead a raga is defined in terms of notes sequence and other features explained in the section 1.3.2 based on which the performer/singer of the raga can improvise. This open ended nature of ICM raga means that a computational recognition and/or identification system would need to address a great deal of complexity than would be typically the case for Western Music (WM)

In the next Section we consider computational musicology methods and approaches for recognition/identification (and indeed generation) of Western music. The Section 1.2 we review the major differences between Indian and Western Classical Music in more details, and explain how approaches used in Western music cannot be directly used for ICM.

\subsection{Computational Musicology and Western Music}

Modern WM history can be traced back to medieval times, with the introduction of Chanting into the Catholic Church services. Western Music then started becoming more of an art form with the advances in music notation. Many forms of popular music have been derived from African-Americans, and their innovations of jazz and blues serve as the basis from which much of modern popular music derives [4].

For any music type, especially Western music, genres are the main top level descriptors used by music dealers and librarians to organize their music collections [8]. Hence there are various approaches made in the western music for genre classification. Commonly used methods are k-nearest Neighbors (KNN), Support Vector Machines (SVM), Gaussian Mixture Models (GMM), Linear Discriminant Analysis (LDA), non-negative Matrix Factorization (NMF), Artificial Neural Networks (ANN) and Hidden Markov Model (HMM) [7].

Other research areas of computational musicology include analyzing the music piece with the help of computer program, segmentation of music pieces based on the rhythm, study of rhythm and meter, the separation of MIDI data into musical voices, Automatically classifying keys and chords using a computer program to process digital data, and computational approach for determining the scale, chord or tonality of a music piece based on the music theoretic notation [7] [ 8].

In the next section we describe the key differences between Western Music and Indian Classical Music, and how these differences necessitate radically different analysis model.

\subsection{Western Music vs Indian Music}

Essential differences between the Indian Classical Music and Western music systems are summarized in Table 1: 
Table 1. Comparison of Western Music and Indian Classical Music [5][ 9]

\begin{tabular}{|c|c|c|}
\hline $\begin{array}{l}\text { Features for } \\
\text { comparison }\end{array}$ & $\begin{array}{c}\text { Indian Classical } \\
\text { Music (ICM) }\end{array}$ & $\begin{array}{c}\text { Western Music } \\
(\mathrm{WM})\end{array}$ \\
\hline $\begin{array}{c}\text { Melody vs } \\
\text { Harmony }\end{array}$ & $\begin{array}{l}\text { Based on Melody } \\
\text { where individual } \\
\text { notes are played in } \\
\text { the given order. }\end{array}$ & $\begin{array}{l}\text { Based on Harmony } \\
\text { where groups of } \\
\text { notes called chords } \\
\text { are played } \\
\text { simultaneously. }\end{array}$ \\
\hline Raga System & $\begin{array}{l}\text { Based on Raga } \\
\text { system which } \\
\text { determines notes } \\
\text { and its sequence. }\end{array}$ & $\begin{array}{l}\text { There is no concept } \\
\text { of raga. Composer is } \\
\text { more constrained by } \\
\text { dominant note and } \\
\text { harmonics. }\end{array}$ \\
\hline $\begin{array}{c}\text { Place of } \\
\text { composition }\end{array}$ & $\begin{array}{l}\text { Based on the } \\
\text { predefined } \\
\text { grammar of a Raga, } \\
\text { the song is } \\
\text { composed by the } \\
\text { singer as part of the } \\
\text { rendition. }\end{array}$ & $\begin{array}{l}\text { A composer first } \\
\text { generates notation } \\
\text { for a tune. The } \\
\text { novice learners } \\
\text { perform this music } \\
\text { piece as per the } \\
\text { notation without } \\
\text { making any changes. }\end{array}$ \\
\hline $\begin{array}{l}\text { Improvisation } \\
\text { of } \\
\text { composition }\end{array}$ & $\begin{array}{l}\text { A musician can } \\
\text { improvise the } \\
\text { composition to } \\
\text { enhance the beauty } \\
\text { and evoke the mood } \\
\text { of raga by } \\
\text { following the } \\
\text { grammar rules of a } \\
\text { raga. }\end{array}$ & $\begin{array}{l}\text { There is hardly any } \\
\text { concept of } \\
\text { improvisation after } \\
\text { the composition is } \\
\text { ready. }\end{array}$ \\
\hline Mood of Song & $\begin{array}{l}\text { Generally has a } \\
\text { principal mood for } \\
\text { a Raga. }\end{array}$ & $\begin{array}{l}\text { Capable of producing } \\
\text { many moods and } \\
\text { feelings in a song. }\end{array}$ \\
\hline Use of drone & $\begin{array}{l}\text { Drone is played in } \\
\text { the background as } \\
\text { constant register of } \\
\text { the tonic note. }\end{array}$ & $\begin{array}{l}\text { Single tonic note is } \\
\text { not required as music } \\
\text { is based harmony. }\end{array}$ \\
\hline $\begin{array}{l}\text { Rhythm } \\
\text { (Taal) }\end{array}$ & $\begin{array}{c}\text { Notes are } \\
\text { played/sung over a } \\
\text { cycle of beats that } \\
\text { repeats itself. It is } \\
\text { important factor to } \\
\text { decide correctness } \\
\text { of song rendition. } \\
\text { So there is great use } \\
\text { of percussion } \\
\text { instruments like } \\
\text { table, dholak, } \\
\text { mrudang and dhol. }\end{array}$ & $\begin{array}{l}\text { Complex beat cycles } \\
\text { are not used for most } \\
\text { music. Beat timing is } \\
\text { important but it does } \\
\text { not determine overall } \\
\text { structure of } \\
\text { played/sung music. }\end{array}$ \\
\hline $\begin{array}{c}\text { Microtones } \\
\text { and Semitones }\end{array}$ & $\begin{array}{c}\text { Indian notes are } \\
\text { divided into } 22 \\
\text { smaller units called } \\
\text { shruties. }\end{array}$ & $\begin{array}{c}\text { Has only } 12 \\
\text { semitones }\end{array}$ \\
\hline
\end{tabular}

As it is clear from Table 1, ICM differs from its Western counterparts and hence the computational musicology methods used in WM cannot be effectively and directly used to analyze or model ICM [15]. Major challenges faced by ICM are its unique Raga system, importance of rhythm and improvisation of composition by the artists.

In Section 1.3, next we introduce the key features of ICM which determines the grammar, which is the rules to determine the structure or rendition of a Raga. This will help provide further insights into the extent to which computational musicology approaches to analyse/model $\mathrm{WM}$ are applicable to ICM. In the section 2 we review and critically evaluate some of major attempts to analyze ICM.

\subsection{Hindustani Classical Music}

\subsubsection{Introduction}

Indian classical music exists in two different forms Hindustani music and Carnatic music, which originated and was primarily practiced in Northern and Southern India respectively. However, both forms of Indian classical music are now practiced and performed in all parts of India. The musical notes of the Hindustani music scale are shadaj (Sa), rishabh $(\mathrm{Re})$, gandhar $(\mathrm{Ga})$, madhyam $(\mathrm{Ma})$, pancham $(\mathrm{P})$, dhaivat (Dh), and nishad (Ni). Collectively these notes are known as Sargam, equivalent to the Solfege of the Western music system.

In Indian Classical Music, tonic scale is required to identify the other swars based on the ratio specified in Table 2. The scale contains only seven natural swars; however, each member may have two different forms except $S$ (the tonic) and $\mathrm{P}$ (the perfect fifth), which exist in only one form. The natural notes are known as shuddha swars, flat notes are the komal swars and the sharp notes are called tivra swars. The different forms of R, G, D, and $\mathrm{N}$ are komal R, shuddha R, komal G, shuddha G, komal D, shuddha D and komal N, shuddha N, respectively. Shuddha $\mathrm{M}$ and Tivra $\mathrm{M}$ are the two variations of $\mathrm{M}$. Thus, the total number of swars or intervals is twelve.

Table 2. Western and Indian notes with frequency ratio [22]

\begin{tabular}{|c|c|c|c|}
\hline $\begin{array}{c}\text { Sr. } \\
\text { no. }\end{array}$ & $\begin{array}{c}\text { Western } \\
\text { Notation }\end{array}$ & Indian Notation & $\begin{array}{c}\text { Frequency } \\
\text { Ratio(natural) }\end{array}$ \\
\hline 1 & $\mathrm{C}$ & $\mathrm{Sa}$ & $1 / 1$ \\
\hline 2 & $\mathrm{C \#}$ & $\mathrm{Re}$ & $16 / 15$ \\
\hline 3 & $\mathrm{D}$ & $\mathrm{Re}$ & $9 / 8$ \\
\hline 4 & $\mathrm{~Eb}$ & $\mathrm{Ga}$ & $6 / 5$ \\
\hline 5 & $\mathrm{E}$ & $\mathrm{Ga}$ & $5 / 4$ \\
\hline 6 & $\mathrm{~F}$ & $\mathrm{Ma}$ & $4 / 3$ \\
\hline 7 & $\mathrm{~F}$ & $\mathrm{Ma}$ & $7 / 5$ \\
\hline 8 & $\mathrm{G}$ & $\mathrm{P}$ & $3 / 2$ \\
\hline 9 & $\mathrm{Ab}$ & $\mathrm{Dh}$ & $8 / 5$ \\
\hline 10 & $\mathrm{~A}$ & $\mathrm{Dh}$ & $5 / 3$ \\
\hline 11 & $\mathrm{Bb}$ & $\mathrm{Ni}$ & $9 / 5$ \\
\hline 12 & $\mathrm{~B}$ & $\mathrm{Ni}$ & $15 / 8$ \\
\hline 13 & $\mathrm{C}$ & Sa' (Next octave) & $2 / 1$ \\
\hline
\end{tabular}

\subsubsection{Features of Indian Classical Music}

It is important to understand that raga is not just a sequence of notes. There are several features that decide the grammar for representation of raga and can be used for raga analysis. Due to these features, various ragas having same set of swars sound differently. Features of ragas are as follows [5]:

- Swars - 12 kind of sound that match the scale are called swar or note. As mentioned in section 4.1 these notes can be in normal, sharp or flat form. Out of 12 notes, 7 are shudh(normal) notes and 4 are komal (flat) swars and 1 tivra (sharp) swar.

- Saptak (Octave) - 12 notes mentioned in table 1 together form the saptak or octave. There are 3 types of saptak: Mandra (lower octave), Madhyam (medium octave), Taar (higher octave). Pitch frequencies of same note in all the three octaves vary. In comparison to Madhyam 
saptak, Mandra saptak has lower pitch frequencies and Taar saptak has higher pitch frequencies.

- Jati - Describes type of saptak. Every raga consists of minimum 5 swars and maximum 7 swars. Based on number of swars used in the ascending and descending order of the raga, the jati of raga is decided. According to the number of swars used technical terms that describe Jati are:

Sampurna - Consists of 7 swars.

Shadava- Consists of 6 swars.

Odhav- Consists of 5 swars.

Considering combination of above terms for ascending and descending order of notes there are nine Jati possible as shown in Table 3.

Table 3. Jati of Raga in Hindustani Classical Music

\begin{tabular}{|c|c|c|}
\hline Sr. No. & Ascending & Descending \\
\hline 1 & Sampurna & Sampurna \\
\hline 2 & Sampurna & Shadava \\
\hline 3 & Sampurna & Odhav \\
\hline 4 & Shadava & Sampurna \\
\hline 5 & Shadava & Shadava \\
\hline 6 & Shadava & Odhav \\
\hline 7 & Odhav & Sampurna \\
\hline 8 & Odhav & Shadava \\
\hline 9 & Odhav & Odhav \\
\hline
\end{tabular}

- Aroha-Avaroha - Aroha is the ascending order sequence of swars that describes how the raga moves in its ascending order. Avaroha is the descending order sequence of swars that describe how the raga moves in descending order.

- Vadi swar (king note) - It is the primary swar of the raga which is struck most frequently in the raga. It helps in evoking the mood of the raga to its fullest.

- Samvadi swar (Queen note) - Samvadi Swar is the second most important swar of a Raga and is the second most frequently struck note in its melodic expansion.

- Varjit swar - These are swars which are regarded as incompatible with the notes of a specified raga. So they are not to be used in the rendition of that particular raga.

- Pakad - Phrases of notes that helps in identifying the raga. It is used by experts for raga identification and also added in the song for enhancing the quality of song.

Example: Pakad for raga Bhupali is:

$\mathrm{P}, \mathrm{Ga}, \mathrm{Dh}, \mathrm{P}, \mathrm{Ga}, \mathrm{Re}, \mathrm{Ga}, \mathrm{Re}, \mathrm{Sa}$

\section{STUDY OF EXISTING WORK IN INDIAN CLASSICAL MUSIC}

Compared with WCM computational musicology research and analysis relatively little work has been done in the field of for ICM. Here we review and evaluate the nature of the most major works done to analyze or model ICM.

\subsection{Searching for common language of ragas}

In 1994, H.V. Sahasrabuddhe and his team had designed a finite automaton model for ICM raga [12]. Their approach was based on the hypothesis that ragas in ICM could be defined in terms of melodic phrases, and that these phrases once identified can be used to compose/generate a ragas. This tool included modules for music composition and printing output. This early attempt met with limited success in terms of rule based generation of ICM/raga. However, it serves to highlight the fact that analysis of raga based on Pattern recognition of melodic phrases would not be sufficient for error free recognition or generation of raga.

\subsection{An Overview of Hindustani Music in the context of computational musicology}

This paper by Rao and Rao [13] is one of the most recent attempt to identify and codify key aspect of ICM (Hindustani and Carnatic) that would be critical in development of computational musicology models of ragas.

As already stated in Section 1.3, this paper highlights the fact that ICM is governed or defined by the raga (ie, sequence of notes and taal (ie, the beat). It further clarifies that ICM performance has three major elements: The primary melody rendered/sung by the singer/performer; the secondary melody which is typically rendered by accompanying melodic instruments (usually string instrument such as veena or sarangi); and the third element is the taal (ie, rhythm) provided by the percussion instruments (ie, tabla).

A performance (or rendition) of a raga in ICM is a blend of sequence of notes which makes up a particular raga (and its mood) and improvisation of the notes - that is, legitimate (rules based) variation of the sequence of notes and variations in the duration of note(s). The rendering/singing of the notes is closely related to as well as determined by the taal (ie, rhythmic structure).

Given this the paper concludes with the valid observation that computational musicology models of raga analysis or recognition would need to take in the account both the notes as well as the critical role of taal. And that given the prevalence of improvisation in a rendition of any raga such a model would need to address this complexity (one that models of Western Classical music don't need to address as we have discussed in Section 1.2).

It also highlights the need and opportunities for models that can enhance the functioning of recognition models designed to assist with retrieval of ICM from online data bases (which for western music are already in wide usage, eg Shazam, Vericast, Gracenote MusicID)

\subsection{Hindustani Raga Representation and Identification: A Transition Probability based approach}

This paper BY Bhattacharjee and Srinivasan [11] describes a transition probability model for identification/recognition of ICM ragas. Mainly is it based on the idea that in any raga the probability for transition between notes in ascending and desending order (ie, aroha-avrohana) is a good determiner of the raga. In many ways this approach is ideal for handling the improvisation aspect of ICM ragas. However, as we have already explained in section 4.2 there are other characteristics of raga which can be considered for its recognition/evaluation/composition.

The authors first manually derived Transition Matrix for 10 ragas by considering detailed analysis of renditions by two singers/musicians for each raga. Each matrix is made up of a 12 columns and rows to represent transition between 12 swars. The transition matrix does not take into the account the octave of the swars; as swars from higher and lower octave are shifted to the middle octave. The probability of transitions for each raga was derived from a training set of 10 clips of 5 seconds. 
The matrix basically predicts the probability of transition from the current note to the next possible note, and it does so for note transition in ascending or descending (aroha-avrohana) order (as these often differ in a raga). When there is conflict in transition possibility; that is when the TPM predicts the same probability for transition to more than 1 note then the ascending or descending direction is taken into consideration and preference is given to the current flowing direction.

Authors generated the aroha-avrohana patterns from the TPM and compared it with the information available through text and literature of ICM such as Bhatkhande Sangeet Shastra or books available from Bharatiya Gandharva Mahavidhyalaya Mandal [19]. They report a 100\% recognition for the set of 10 ragas. However, it is arguable that this model has limited application for two reasons: First the manual generation of TM would not feasible for all ragas and/or renditions/performances. The model needs to be tested with wide range of songs sung by multiple artists for more accurate results.

A more robust model could be developed if the transition matrix would also consider other features of ICM mentioned in section 1.3.2. Similarly the decision to focus on only the middle octave (and shifting the notes from higher and lower octave to middle octave) means that critical information is lost as at times aroha-avarohan sequence can change with singer's shift between octaves: For instance a sequence Sa(Middle) $\mathrm{Ga}$ (Lower) is considered to be avarohan.

Study also represents the Relationship between 2 ragas by calculating the Euclidean distance between ragas using TPM. With Euclidean distance and occurrence of swars for ragas authors have tried to represent similarities between ragas; as similar ragas would have greater usage of same notes which results in higher co-efficient correlation between ragas. According to authors, this information can be further used for identifying the tonal hierarchies.

\subsection{Harmonium Raga Recognition}

This paper by Pandekar et al [15] describes a model that automatically recognizes notes played on a harmonium, and then determines the raga being rendered by the notes.

The model is based on Spectral Flux [21] and Fundamental Frequency Estimation algorithms which are typically used for onset and end point detection of notes [20]. The process for note identification starts with the pitch frequencies extraction. These extracted frequencies are compared with the database which contains all the possible swars with their corresponding values of pitch frequencies. Tolerance permitted for this process is $+2 \mathrm{~Hz}$ to $-2 \mathrm{~Hz}$.

For raga recognition the authors developed a database of all grammatically valid note combination for eight different ragas. This database was hand crafted from in depth analysis of harmonium music recordings of professional musicians. The identified notes (sequence) are compared with the raga database with a simple template matching algorithm.

The model performed very well with $100 \%$ accuracy in terms of recognition of the four ragas which consist of 25 prerecorded standalone harmonium performances. However, this success needs to take in the account that identification of notes played on a harmonium is relatively straightforward because frequencies of notes played by an instrument do not fluctuate as would be case for vocal rendition.

The model has limited applicability as it is designed to recognize note sequences of a subset of eight raga. It is highly likely that extending this model to more ragas would probably necessitate major changes in the raga database. The number of possible grammatical sequences would probably increase in a non-linear manner, thus increasing complexity and affect computational as well as predictability performance.

This approach would also prove to be inappropriate for identifying different ragas with the same set of notes or sequences (ie, phrases) with very minute differences. For example, raga "Bhimpalasi" and "Dhanashree" have the same notes and sequence. Hence in some respect the grammar of the phrases (ie, note sequences) is very similar. The only difference being that in raga "Dhanashree" the use of note " $\mathrm{Pa}$ " is increased compared to "Ma" (and vice versa with respect to raga "Bhimpalasi"). It is unlikely the proposed model would be able to distinguish between renditions in these two ragas without a simultaneous increase in computational complexity.

Desptite the limitations of general applicability of the proposed model this is a good implementation of note identification from raw audio input. The note recognition module is good at extracting key features of the sound wave file and segmentation into swars (ie, notes) with nearly $100 \%$. It serves to highlight the critical importance of fast and accurate note recognition module (algorithm) for a tutoring system for the novice learner and especially if this is achieved in real time.

\subsection{Tansen: A system for Automatic Raga Identification}

-In this paper Pandey et al [16] have proposed a system for Automatic Raga Identification. They have implemented such a system for recognition of two ragas: "Yaman Kalyan" and "Bhupali". The system basically has three modules. The first one is designed to take audio inputs (ie, as sound file) and output note transcription. Pitch listing with window size of 0.01 and 0.05 is generated using PRAAT software. Note transcription module uses two heuristics: Hill Peak Heuristics and Note Duration Heuristics. Hill Peak Heuristics evaluates the pitch graph for hill/ peak based on which it helps to identify the notes in an audio input. Note Duration Heuristics is designed to determine the likely duration of the note (beyond which the sound segment is reconsidered by the Hill Peak Heuristic). For purpose of ICM authors have fixed the duration at $25 \mathrm{~ms}$ per note.

The second module is designed to identify the basic (or Plain) raga. This uses the string or pattern matching approach, which is this case is a Hidden Markov Model (HMM). The authors used the Baum-Welch learning algorithm to train HMM with set of note sequence of a particular raga. So each raga has a separate HMM. Essentially the HMM models represent the transition probability of notes or phrases of a raga which the authors refer to as the "semantic" of a raga. However, this model was then used to predict raga type with a test set, achieving an average accuracy of $77 \%$ for plain raga identification.

The $77 \%$ accuracy was improved by then considering a specific feature of raga, ie, pakad which is one of the key determiners of the nature and style of Raga and can be considered as key aspect for improvisation of rendition. This was achieved by passing the note transcriptions through Pakad Matching module. Authors have devised two ways for matching of the pakad with input string of notes: $\delta$-occurrence with $\alpha$-bounded gaps and n-grams matching. $\delta$-occurrence with $\alpha$-bounded gaps algorithm works on the assumption that a piece of pakad say $(t)$ can be declared present in the sample 
say (p) if all the notes of $t$ exists in the specific bounds. Whereas n-grams matching algorithm works on capturing the appearance of pakad within a raga by counting the frequencies of appearance of successive n-gram of the pakad. Average result of raga identification with pakad matching was $87 \%$.

While the authors report very impressive results particularly with respect to raga recognition base on pakad matching, this performance is limted to only two ragas (ie, "Yaman Kalyan" and "Bhupali"). This model is further constrained by modelling renditions of both ragas in only one octave/scale (ie, G sharp). This is somewhat restrictive as singers are likely to use other scales.

The note transcription module is computationally complicated as the methods used in it require different sampling rate. Hill Peak Heuristics requires low sampling rate whereas Note duration heuristics requires high sampling rate.

The paper emphasizes on use of HMM for plain raga identification and pakad matching algorithm to improve the results. To achieve the goal of raga identification, authors have used probabilistic automata constructed on the basis of the notes unlike former system which generally used low level features like spectral centroid, spectral roll off, spectral flux, Mel Frequency Cepstral coefficients etc. for classification.

\section{SUMMARY}

The primary objective of the study was to understand the Raga system and its features which can be used to define Raga structure. Computational musicology in context of ICM has 3 major research areas which are as follows: Music composition, Music Recognition/Identification and Music Improvisation. ICM is very open-ended and flexible, which makes it complex to be represented in computational model. Though study suggests that statistical information imbibed in swar sequence is very important factor for raga analysis.

Music composition are rule based systems modelled using the existing rules and features of ICM mentioned in section 1.3.2. However systems available for music composition work more like sequence generator considering the features of raga. But raga is more than just a set of notes and rules, and artistic sense of raga cannot be captured only by form of rules. Random sequence of notes generated by music composition software cannot always generate beautiful sound pattern. This is the reason that alternate technique of grammars and finite state automata can be used for music composition. Also apart from the features mentioned in section 1.3.2, other features like fade-ins/ fade-out, loudness and duration of notes can improve the quality of composed music. Most of the systems are built using Hidden Markov Model (HMM) as it is ideal for generating random sequence of notes considering small set of input. It would be interesting to study how hybrid systems can be generated using HMM and other genetic algorithms and does it help in improvising the quality of composed music.

Music Recognition systems majorly target the identification of raga from a song rendition. It is a complex task as it further combines research areas like frequency extraction, automatic note transcription and pattern matching (for phrase identification). Phrases being one of the important feature for raga identification leads to the usage of several pattern matching algorithms. However, phrases being present in any part of the composition and in any order makes simple pattern matching ineffective for obtaining accurate results. Also it would become computationally inefficient to handle the large corpus of phrases of different ragas and comparing them with inputted songs. Also, similarities between ragas can add to the complexity as ragas having similar set of notes may generate same sequences with minute differences which can be difficult to be identified.

Other interesting research gap is the automatic segmentation and labeling of melodic motifs from audio recordings which can be important for raga identification applications as well as Music Information Retrieval systems. Music Information Retrieval is response to the rapid growth of digitized music. The availability of vast digital audio archives has opened up several prospects of research. There are software like Shazam, Soundhound, musixmatch and Soundtrack used to retrieve the audio file from large music database.

Datasets used for testing the models are very constrained either in number of ragas supported or constrained to performances of 1 or 2 artists. As more number of ragas are considered in the system, it would increase the computation complexity.

While we have restricted our discussion to computational aspects of melody, there is equal scope for computational research in the rhythm aspects. The hybrid system considering melody and rhythm would give much better results there by considering taal and tempo for audio segmentation.

As we move from novice learners to experts there are other features of raga such as Alap (Section to show individuality and flavor of raga), taan (a rapid sequence of notes), meend (slide from one note to other which might also include varjit swars of a raga), laykari (introduction to rhythmic variations) etc. which would add to the complexity of the process of composition as well as recognition.

\section{TUTORING SYSTEM FOR INDIAN CLASSICAL MUSIC}

ICM follows the guru-shishya methodology for learning music. In ancient times, student stayed with guru, accompanied him for musical performances, practiced (riyaz) in his presence and learned music. In today's world it is difficult to follow the same routine. As ICM is very rich with many ragas having the similar structures with minor differences in their rendition, it is difficult for a novice learner or listener to distinguish between the ragas. Also unlike western music, ICM along with the 12 major notes also has the concept of shrutis (minor notes) and gamakas (ornamentation) which makes the process of distinguishing the notes (swars) difficult. So, for a student practicing the raga without the presence of guru, an evaluation system which can check how accurately the raga is performed can be very helpful. It can work like a feedback or tutoring system for beginners [15].

Improvisation is a process done after a composition is ready or at times improvisation goes along with the composing process. An artist of ICM should not be confined into strict pieces of ideas; rather he has to wander to be explorative to produce artistic pleasures with his talent and imaginary powers. Improvisation process can also include the guidance provided to novice users for the mistakes made in singing the notes. Even if notes are sang correctly, based on the features of ICM (aroha-avaroha, vadi swar, samvadi swar, varjit swar, pakad), there is possibility of improvising the song by changing the sequence of notes, increasing the usage of king and queen notes and also by integrating the "pakad" phrases in the raga. The most aesthetically important aspect of good improvisation in raga is maintaining the rasa (mood) of raga while making the changes. 


\section{CONCLUSION}

From the above overview of recent research in computational musicology with respect to ICM, it is clear that the focus has been largely on recognition of music (or raga); that attempts at computer based models to recognize patterns (of notes, usually) and motifs based on which the music is identified in terms of raga or composition. . While such models are appropriate it can be argued that they have a major limitation: Given the key importance of improvisation in ICM computational musicology models of ICM need to be able to evaluate the nature of improvisation (and therefore by extension judge the quality of music being sung or played).

A general purpose system (or computational model) to evaluate ICM would pose a major challenge. This is because of the huge range of variability between and within ragas (as is evident from overview of ICM in Section 2). Hence it would be more appropriate to develop a more constrained model for quality evaluation of specific (individual) ragas and that too limited to specific instruments or voice. Or such an evaluation model can be constrained in terms of range and scope: for example, a model designed to evaluate songs in sub-set that have a fairly simple composition and duration. Such songs are often the ones that novice of vocal ICM use for practice, that is to learn to enunciate the notes as well as improvise (but in a fairly limited manner). This sort of computational model then can be used as part of tutoring system for novice learner, whereby the system can evaluate the songs such by the learner and based on which can provide feedback of two types: highlight the wrong notes as well as suggest ways in which they could improvise at key points in the song that would enhance the quality of their rendering/output. Such a system for evaluation and providing improvisation feedback would incorporate both elements of grammar of ICM as well as basic (or underlying) rules of for acceptable improvisation.

\section{REFERENCES}

[1] Volk, Anja, Frans Wiering, and van P. Kranenburg. "Unfolding the potential of computational musicology." Proceedings of the 13th International Conference on Informatics and Semiotics in Organisations. 2011

[2] Coutinho, Eduardo, et al. "Computational musicology: An artificial life approach." Artificial intelligence, 2005. epia 2005. portuguese conference on. IEEE, 2005.

[3] Juan R. Rabunal, Julian Dorado, Artificial neural networks in real life applications, Idea Group Publishing, Yurchak Printing Inc.

[4] Western Culture, Available at: http://en.wikipedia.org/wiki/Western_culture\#Music , Accessed on 6/3/2015.

[5] Indian Classical Music and Sikh Kirtan, Available at: http://fateh.sikhnet.com/sikhnet/gurbani.nsf/d9c75ce4db 27be328725639a0063aecc/085885984cfaafcb872565bc0 04de79f!OpenDocument , Accessed on 11/2/2015

[6] Computational Musicology, Available at: https://en.wikipedia.org/wiki/Computational_musicology , Accessed on 5/2/2015
[7] Agarwal, Parul, Harish Karnick, and Bhiksha Raj. "A Comparative Study Of Indian And Western Music Forms." ISMIR. 2013.

[8] Scaringella, Nicolas, Giorgio Zoia, and Daniel Mlynek. "Automatic genre classification of music content: a survey." Signal Processing Magazine, IEEE23.2 (2006): 133-141.

[9] Bajpai, Garima. "Music and Personality."

[10] Jairazbhoy, Nazir Ali. The rāgs of North Indian music: their structure and evolution. Popular Prakashan, 1995.

[11] Bhattacharjee, A. R. I. N. D. A. M., and N. A. R. A. Y. A. N. A. N. Srinivasan. "Hindustani raga representation and identification: a transition probability based approach." IJMBC 2.1-2 (2011): 66-91.

[12] Sahasrabuddhe, H. V. "Searching for a Common Language of Ragas": Proc."Indian Music and Computers: Can'Mindware'and Software Meet (1994).

[13] Rao, Suvarnalata, and Preeti Rao. "An overview of Hindustani music in the context of computational musicology." Journal of New Music Research 43.1 (2014): 24-33.

[14] Ganguli, Kaustuv Kanti, and Preeti Rao. "Tempo dependence of melodic shapes in hindustani classical music." Mana 160.7.3 (2014): 1-148.

[15] Pendekar, Rajshri, et al - here you have to have the authors names. "Harmonium Raga Recognition." International Journal of Machine Learning and Computing 3.4 (2013): 352.

[16] Pandey, Gaurav, Chaitanya Mishra, and Paul Ipe. "TANSEN: A System for Automatic Raga Identification." IICAI. 2003.

[17] Das, Dipanjan, and Monojit Choudhury. "Finite state models for generation of Hindustani classical music." Proceedings of International Symposium on Frontiers of Research in Speech and Music. 2005.

[18] Sinith, M., and K. Rajeev. "Hidden markov model based recognition of musical pattern in south Indian classical music." IEEE International Conference on Signal and Image Processing, Hubli, India. 2006.

[19] Akhil Bharatiya Gandharva Mahavidhyalaya Mandal, Available at: http://abgmvm.org/home.html, Accessed on 17/2/2016.

[20] Fundamental Frequency Estimation, Available at: http://recherche.ircam.fr/anasyn/roebel/amt_audiosignale /VL5.pdf, Accessed on 17/2/2016.

[21] Onset Detection: Onsets and Spectrul Flux, Available at: http://www.badlogicgames.com/wordpress/?p=161, Accessed on 18/2/2016.

[22] Shruti, Available at: https://en.wikipedia.org/wiki/Shruti_(music), Accessed on $11 / 8 / 2017$. 